\title{
HACIA UN NUEVO CONCEPTO DE APRENDIZAJE DE LAS LENGUAS
}

\author{
Begoña Souviron López \\ Departamento de Didáctica de las Lenguas, \\ las Artes y el Deporte \\ Facultad de Ciencias de la Educación \\ Universidad de Málaga \\ http://dx.doi.org/10.18778/8220-201-4.30
}

\section{Resumen}

Como en la actualidad la alfabetización digital implica el uso de recursos tecnológicos y el desarrollo de la competencia comunicativa para exponer y compartir el aprendizaje, aprovechamos este espacio para tomar en consideración aspectos didácticos que tienen que ver con las condiciones en las que se dan los procesos de aprendizaje y proponer nuevas estrategias metacognitivas que incluyen la evaluación de las condiciones en las que se produce en el seno de grupos de formación de profesorado de lenguas.

Palabras clave: Estrategias de aprendizaje de lenguas, aspectos metodológicos de la enseñanza de lenguas, aprendizaje entre iguales.

\section{1. \\ El cambio de paradigma didáctico}

La alfabetización digital implica el uso de recursos electrónicos y el desarrollo de la competencia comunicativa para transmitir de qué modo esos recursos contribuyen a la construcción social del aprendizaje en la sociedad del conocimiento. Ese proceso, que la mayoría de las veces se estructura como aprendizaje cooperativo, 
requiere del desarrollo y la puesta a punto de la competencia digital y la competencia literaria, en su más amplio sentido, como competencia cultural y mediática, que se desarrollan de forma paralela constituyendo su paulatino dominio el consiguiente refuerzo de ambas. Por otra parte, en un mundo globalizado, donde el carácter de la información es predominantemente mediático, el "médium" o "transfer" es visual y dinámico como lo demuestra la proliferación y la construcción de la propia imagen en plataformas como Instagram, YouTube o Faceboook y la relevancia de la fotografía como medio para visibilizar el producto sobre el que desarrollamos nuestro trabajo, e incluso la evidencia de los resultados alcanzados.

Espacios conceptuales nuevos como los de interculturalidad e interdisciplinaridad y ejes de conocimiento como el de transversalidad o interseccionalidad nos permiten acceder desde diferentes perspectivas a distintos estadios de la construcción social del aprendizaje a la vez que propician nuevas formas de crear una conciencia de sí. En ese sentido es interesante examinar y plantear la cuestión de las condiciones que propician el aprendizaje entre iguales, en nuestro caso entre mujeres en diferentes etapas educativas desde la concepción feminista del sujeto que no puede separarse de la formación de un sujeto de conocimiento y acción.

Como destaca Churches (2008) en la base de la pirámide del Aprendizaje, de la Taxonomía de Bloom, se halla el Conocimiento, entendido en nuestros días no ya como acumulación de contenidos, sino como la capacidad de localizar, acceder, descubrir y transmitir determinados saberes, procedimientos y actitudes. En el vértice de dicha pirámide se sitúa el producto del aprendizaje manifiesto, no sólo lo aprendido, sino la explicación del proceso que se ha seguido hasta alcanzar dicho aprendizaje y la metodología mediante la cual se ha hecho posible. Por eso, en ese mismo vértice encontramos la Evaluación, que incluye la comparación de ideas, así como las ventajas e inconvenientes de los procesos desarrollados y la facultad de estimar, valorar y recomendar a partir de la experiencia acumulada en el itinerario. Ya en el centro de la pirámide localizamos los procesos de aprendizaje más significativos como son: la Comprensión, la Aplicación, el Análisis y la Síntesis. De ese orden llama asimismo 
la atención el lugar predominante concedido a la fase de Aplicación porque en nuestro mundo no se concibe el conocimiento significativo sin una utilidad práctica en la realidad cuya influencia ha de ser manifiesta y argumentada. Posteriores fases de Análisis y Síntesis, sobre las que volveremos más adelante, se producen respecto a percepción y comprensión de la realidad a la vez que consideran la eficacia o viabilidad del grado de aplicación de los procesos.

En este sentido tenemos que señalar la importancia que adquiere la revolución feminista del conocimiento. Ya desde la teoría crítica un sector del feminismo alemán de los 80 propuso frente al absoluto de la razón instrumental un sujeto que cultivara la razón sensual lo que determinó la construcción de la teoría de la racionalidad y la acción social. Así, autoras como Neus Campillo (2005) destaca la aportación que en esta dirección realizó Sheila Benhabid al reclamar la necesidad de tener en cuenta el subtexto de género y la consideración de "otro concreto" que demanda la atención de un "yo situado" en una determinada articulación discursiva y concluía exponiendo la teoría de la racionalidad y la acción social, así como la necesidad de plantear mejores fórmulas para subvertir la jerarquía de poder de lo masculino sobre lo femenino.

Una metodología basada en la citada taxonomía y desde la perspectiva aludida se caracteriza en breves rasgos por:

- El uso de conceptos conocidos para crear nuevas ideas y dar voz a las mujeres en el ámbito experimental.

- El diseño de intervenciones que promuevan la adopción de modelos y sistemas de aprendizaje que favorezcan el desarrollo de competencias metacognitivas según los estilos de aprendizaje de iguales.

- El desarrollo de estrategias que fomenten la competencia de "aprender a aprender" en el seno de grupos heterogéneos y homogéneos.

- La identificación de modelos que promuevan metodologías basadas en el planteamiento de situaciones de aprendizaje a las que hay que dar respuesta desde distintos ámbitos de trabajo cooperativo.

- El análisis de estrategias que permitan desarrollar los puntos anteriores. 
En la actualidad necesitamos enseñar para aprender a explicar cómo hacemos las cosas y de qué manera han sido y son pensadas. Pero a la hora de alcanzar este propósito lo primero que debemos promover es una fase previa de Acomodación donde predomine el sentir y el hacer, porque haciendo experimentamos de manera activa y podemos reflexionar para conseguir que ambos procesos converjan en la misma dirección. Cuando desarrollamos una perspectiva crítica al mirar y sentir la realidad como objeto de conocimiento en sus distintas manifestaciones y adoptamos una actitud de divergencia para, a la luz de ésta, volver a reflexionar sobre aspectos inéditos de la misma, nuestra percepción se convierte en un pensar de nuevo la realidad y pasamos a desarrollar la fase de Asimilación. Así pues, desde estas premisas, apelamos a una cita que puede resituarnos respecto a nuestras concepciones de Aprendizaje: "In relation to learning, ontology trumps epistemology. That is today, the students's being in the world is more important for their learning than their interests in developing knowledge and understanding in a particular field (Barnett, 2007: 6).

2.

\section{Pensando como se produce el aprendizaje}

Moseley (2005) propone con otros que el pensamiento como proceso, en el sentido en que concebía el término Dewey (1989), está encadenado y guiado por el cuestionamiento de alguna instancia cuyo fin es el de alcanzar conclusiones. En la base de la racionalidad y de la acción sensible, la experiencia se relacionaría igualmente con el concepto de metacognición, entendiendo por éste la manera en la que pensamos sobre cómo se produce nuestro aprendizaje, cómo identificamos y progresamos en los procesos que lo articulan y estructuran y, sobre todo, nos sentimos en ese proceso.

La metacognición implica dos dimensiones: la capacidad de conocer el funcionamiento de nuestro propio pensamiento y el 
uso de los recursos propios del conocimiento para ser aplicados en el aprendizaje o solución de problemas en otros ámbitos.

Muchas intervenciones en clase están marcadas por tendencias basadas en teorías que proceden de esos marcos de investigación como sucede en el caso de la Autorregulación, que contribuye a ayudar a las alumnas y alumnos a desarrollar una actitud positiva ante el aprendizaje, haciéndoles confiar en que su implicación cognitiva y emocional les llevará a superar con éxito el proceso en el que se hallan inmersos. La Autorregulación como proceso fundamental del aprendizaje implica por tanto la asunción de un comportamiento que afecta a los ámbitos cognitivo, afectivo, motivacional y actitudinal y permite a cada individuo adecuar sus acciones a la consecución de determinados objetivos, contribuyendo así en la medida de lo posible a influir en la realidad circundante.

Los programas basados en el desarrollo de estrategias de pensamiento, tanto metacognitivas como cognitivas, fueron desarrollados en su día por Ashman y Conway (1990 apud Moseley, 2005: 24) y se basan en los siguientes principios:

- Los fundamentos de estos programas son:

- La metacognición

- El desarrollo del Pensamiento crítico

- El fomento del Pensamiento creativo

- La definición y articulación de los Procesos cognitivos

- La práctica de destrezas integradas

- La Comprensión de las diferentes funciones de los contenidos y roles del conocimiento

Después de Vygotski o Piaget las tareas son concebidas para facilitar las acciones y procedimientos que ha de seguir el aprendiz hasta desarrollar la capacidad de actuar de manera autónoma (Rogoff, 1990). Por su parte, ya la teoría del aprendizaje mediatizado de Feurestein (1979 apud Orru, 2003: 44) postulaba una serie de fundamentos en la interacción del profesor con el alumno que no siempre tenemos en cuenta en las aulas a la hora de entablar un diálogo productivo entre iguales; si bien en los programas de coeducación y protección contra la violencia de género tienen su lugar preponderante. 
Intencionalidad, Reciprocidad; Coparticipación; Significado, Individualización, Trascendencia, Planificación de Objetivos, Competencia, Retos, Autorregulación y control de comportamientos y Automodificación son instancias de este modelo que propone en su programación el desarrollo de destrezas mentales y de estrategias cognitivas. Las primeras se refieren a las operaciones metacognitivas: Discriminar, Aprender conceptos, Usar reglas, Usar reglas de orden mayor y procedimientos. Las estrategias cognitivas, por su parte, resuelven procesos o problemas como el del control ejecutivo.

Por su parte, otras taxonomías como las de Stahl y Murphy (1981) (apud Moseley, 2005: 80) se manifestan útiles para diseñar aprendizajes y proponen 21 procesos mentales: Asociación, Clasificación, Combinación, Comparación, Condensación, Conversión, Descripción, Designación, Discriminación, Extensión, Extracción, Interpretación, Organización, Proposición, Reconciliación, Selección, Separación, Traducción, Utilización, Valoración y Verificación.

Rebeca Oxford (2011) en su programa específico de producción de estrategias de autorregulación para el aprendizaje de las lenguas, apela a la necesidad de relacionar las estrategias con las áreas específicas para observar cómo se sienten los alumnos cuando las ponen en práctica. A tal efecto recomienda desplegarlas en situaciones de aprendizaje concretas, teniendo en cuenta las creencias, las actitudes y la motivación, así como las dimensiones socioculturales que juegan a favor de la interacción en contextos de comunicación y cultura.

El aprendizaje autorregulado comprende procesos que marcan objetivos, atienden a la instrucción, usan estrategias afectivas, seleccionan información para ser recordada, establecen una dirección efectiva del espacio y el tiempo, producen un ambiente de trabajo creativo y productivo a la vez, usan los recursos, recaban orientación y ayuda, mantienen argumentos positivos sobre la propia experiencia y capacidad, así como sobre el valor del aprendizaje y los elementos que influyen en él, y la toma de conciencia en relación a nuestros esfuerzos y éxitos (Schunk y Ertmer, 2000: 631). 


\section{Estrategias y metaestrategias}

Las estrategias y las metaestrategias interactúan ya que estas últimas incluyen estrategias metacognitivas, metaafectivas y metasociales que afectan a las relaciones del grupo en el trabajo cooperativo y repercuten en el tipo de metaconocimiento: personal o de grupo, enfocado a la tarea o al proceso global. Todos ellos contribuyen al conocimiento condicional (cuándo, por qué, y dónde usar determinada estrategia) (Oxford, 2011: 21).

Las estrategias cognitivas inducen a usar los sentidos para comprender y recordar e incluyen la activación del conocimiento, el razonamiento, la conceptualización detallada, que integran análisis y comparación; una conceptualización más amplia, que abarca la síntesis y la sumarización, además de la predicción e hipótesis. Las estrategias afectivas incluyen: activación de creencias, emociones y actitudes, generación y mantenimiento de la motivación. Por último, las estrategias interactivas sociales contemplan la relación entre aprendizaje y comunicación, salvando lagunas de conocimiento mediante otros recursos comunicativos en diferentes contextos culturales o entre identidades (Oxford, 2011: 24).

Las estrategias no pueden ser conocidas de manera unitaria, sino como un conjunto de propiedades, factores y actitudes que se combinan. Ejemplos de estrategias y de tácticas que hacen posible su mejor comprensión son, por ejemplo:

- Ir más allá de los datos observables para predecir resultados a partir de un paradigma cuya estrategia podría pertenecer a la táctica de adivinar el concepto a partir del contexto de lectura recurriendo al propio vocabulario para buscar el sentido.

- Obtener y planificar recursos: ir al diccionario para buscar lo que no se entiende.

- Planificar pidiendo ayuda y estableciendo previamente lo que deseamos hacer, incluso partiendo del modelo de una compañera o compañero para ampliarlo luego.

- Conceptualizar o abstraer, representar mediante mapas, gráficos o dibujos. 
- Conceptualizar materializando, sintetizando y mostrando los componentes.

- Prestar atención a cómo hacer las cosas para superar posibles dificultades.

- Interactuar para aprender y comunicar preparando juntos un examen.

- Activar recursos emocionales, creencias o actitudes, recordando experiencias de aprendizaje significativas que refuercen el aprendizaje.

- Razonar mediante deducciones animándose a establecer hipótesis, por ejemplo: si esto es así....

- Activar el conocimiento mediante técnicas de braimstorming para iluminar y facilitar la exposición o solución de un problema.

- Superar la falta de conocimiento dando rodeos hasta dar con la solución (Oxford, 2011:33).

Es preciso conocer el estilo de aprendizaje dominante para usar las estrategias más adecuadas. Así, según el aprendizaje se produzca en el dominio de lo sensorial, lo social o lo procesual, aparecen estilos diferentes como el visual, auditivo o quinésico; extrovertido o introvertido; analítico, sintético, secuenciativo, intuitivo o abstracto, orientado siempre a la solución inmediata o abierto.

Oxford (2011: 54) remite a la obra de A. Leontiev cuando propone actividades encadenadas que dan lugar a una táctica propia de una estrategia de rango superior ya sea metacognitivo, cognitivo o afectivo. En esas actividades intervienen: el sujeto, el objeto u objetivo, la acción, las condiciones y las operaciones o maneras en las que se manifiesta la acción, determinada siempre por su contexto.

Las estrategias metaafectivas prestan atención al ámbito afectivo para planificar la acción, obtener recursos, causar afecto, implementar planes de trabajo que se centren en las emociones afectivas, usar estrategias para que fluya la afectividad, liderar el afecto y evaluar las respuestas (Oxford, 2011: 65).

Por su parte Wallace (2012) examina las condiciones de aprendizaje y promueve una serie de fases que están integradas en el Sistema de TASC: Thinking Actively in a social context como son: 
- Organizar, exploración sistemática, cuestionamiento de aspectos y reconocimiento del problema.

- Identificar, buscar información adicional, explorar los objetivos, cuestionarse las necesidades, representar la información.

- Generar, producir ideas, consultar con otros, comparar opciones.

- Decidir, tener en cuenta las consecuencias, priorizar, hacer una selección operativa, mostrar ejemplos, planificar pasos y monitorizar.

- Implementar, manifestar el progreso monitorizado, comprobar la eficacia, considerar alternativas.

- Evaluar, la consecución de objetivos, la eficacia de las estrategias personales y de grupo.

- Comunicar, justificar las decisiones, evaluar la evidencia de la información, interacción e intercambio de ideas y explicitar los resultados.

- Aprender de la experiencia, analizar y reflexionar sobre el proceso de solución de los problemas.

- Comparar el presente con el pasado, revisando los procedimientos.

- Generalizar y transferir resultados. Ligorio (2013) precisa una serie de condiciones a partir de las cuales el aprendizaje será significativo:

- Facilidad para reconocer fenómenos esenciales.

- Rapidez y oportunidad para manejarse con los tiempos.

- Exactitud a la hora de diseñar y estructurar el plan de trabajo con cuestiones evocadoras y claras, ilustradas con imágenes significativas con el uso de un lenguaje preciso pero también figurativo.

- Visibilidad en ambos sentidos, de la palabra a la imagen y viceversa para potenciar la imaginación al servicio de la pedagogía.

- Multiplicidad a la hora de tratar los hechos o modelos, así como las consecuentes metodologías a fin de pensar y expresar la complejidad global de la realidad mediática. 
A la pregunta de qué entendemos por aprendizaje creativo, Thomson y Sefton Green (2011) respondieron que es aquél que permite a los alumnos desarrollar sus propias ideas y cultivar el entusiasmo, facilitando diferentes soluciones a posibles problemas que puedan surgir en las respectivas fases del proceso. La mayoría de los proyectos de los que dan cuenta estos autores tiene que ver con el conocimiento adquirido mediante Descubrimiento, donde se dan la mano la experiencia estética y la consideración de la misma, así como su efecto artístico y sentido pedagógico.

La capacidad para implicarse en procesos creativos de pensamiento que potencian el aprendizaje determina lo que Pei Ling Tan (2008: 115) ha llamado cognitive playfulness. Ella estudia a aquellos individuos que tienen una predisposición para la curiosidad, la inventiva, y el deseo de poner en juego nuevas ideas y proyectos, el deseo de innovar o aprender de modo individualizado, y promueve una dinámica con posibles medidores de evaluación a base de ítems como: "Me siento realizado cuando":

- Compruebo que he superado determinadas barreras en el proceso de aprendizaje.

- Cuando veo que lo que he aprendido me sirve fuera del centro.

- Cuando soy capaz de evaluar lo que he aprendido.

- Cuando puedo expresarme sobre el desarrollo de mi aprendizaje.

- Cuando me siento responsable y creador de mi proceso de aprendizaje.

Como afirma Coll (2007: 34-39), los aprendizajes deben ser transferibles y aplicables a otros contextos, sobre todo a la vida real donde siempre aparecen problemas y conflictos nuevos. Esto implica la identificación, selección, caracterización y organización de los aprendizajes escolares, con la intención de que se produzca la transferencia de lo aprendido desde una situación concreta en el aula a otras de la vida real en diferentes contextos sociales. El término Competencia, no exento de polémica según el autor, demanda innovación social, educativa y atención personalizada y equivale a capacidad, atributo, habilidad, destreza. A veces designa repertorios de acciones aprendidas en sus contextos que hacen a las personas eficaces en una determinada situación para resolver problemas. 
El aprendizaje colaborativo se entiende a este efecto, como una tarea compleja donde se negocian y comparten significados para dar solución a un problema, crear o producir algo nuevo y diferente. Sin embargo, los expertos difieren tanto en la descripción de los mecanismos propuestos como respecto a la influencia social (conflicto cognitivo entre iguales - o heterogéneos- versus apoyo de otros más expertos). De la misma manera, disienten sobre los procesos mediante los cuales los individuos se benefician de la relación social (interiorización de la actividad individual externa versus apropiación personal de la actividad compartida).

Habida cuenta de las circunstancias, podemos resumir que el aprendizaje dialógico demanda multiplicidad y complejidad a la hora de extraer los aspectos esenciales; atiende al panorama sociocultural y sitúa simultáneamente los fenómenos. Asimismo este tipo de aprendizaje tiene en cuenta los aspectos colectivos e individuales para evaluar las tendencias y las excepciones tanto en la realidad empírica como en el campo de las representaciones simbólicas. Así establece y observa opiniones diversas respecto a los hechos analizados, teniendo en cuenta los elementos materiales y los valores inmateriales que participan en el diálogo según las coordenadas de espacio y tiempo. El aprendizaje dialógico permite, por añadidura, que afloren otros elementos o categorías que lo hacen efectivo respecto a los conceptos que han de ser aprendidos y respecto al desarrollo personal de los individuos que los protagonizan.

\section{Referencias bibliográficas}

Barnett, R. (2007), A will to learn. Being a student in an age of uncertainty, Berkshire: Open University Press, 6.

Campillo, N. (2005), "Critical Feminism. Citizenship and Political Subject", en E. de Sotelo (ed.), New Women of Spain: Social-Political and Philosophical Studies of Feminist Thought, Münster: Lit Verlag, 78-87.

Churches, A. (2008), “Taxonomía de Bloom en la era digital” [en línea] <http://eduteka.icesi.edu.co/pdfdir/TaxonomiaBloomDigital.pdf $>$ [fecha de consulta: 30.03 .2020$]$. 
Coll, C. (2007), "Las competencias en la educación escolar: algo más que una moda y mucho menos que un remedio", Aula de innovación educativa, Vol. 161, 34-39.

Dewey, J. (1989), Cómo pensamos: nueva exposición de la relación entre pensamiento y proceso educativo. Barcelona: Paidós.

Feuerstein, R. (1979), The Dynamic Assesment of Retarded Performers, The Learning Potential Assesment Device, Theory, Instruments, and Techniques. Baltimore, University Park Press.

Ligorio, B. (2013), "Dialogical learning and dialogical self. Two stories and many interplays”, en J. Valsiner y N. Bohr (eds.), Advances in cultural Psychology: Constructing Human Development, Charlotte NC, XIII.

Moseley D. et alii. (2005), Frameworks for thinking. A Handbook for Teaching and Learning, Cambridge: University Press.

Orru, S. (2003), "Reuven Feuerstein y la teoría de la modificabilidad cognitiva estructural", Revista de Educación, 332, 33-47.

Oxford, R. (2011), Teaching and Researching language: Learning Strategies, Essex: Pearson Education.

Pei Ling Tan, J. y Mc Millan, E. (2008), "Cognitive playfulness, creative capacity and generation C learners", Cultural Science, 1 (2), [en línea] <http://doi.org/105334/csci.12> [fecha de consulta: 30.03 .2020$]$.

Schunk, D. et alii. (2000), "Self regulation and academic learning: self-efficacy enhancing interventions", en M. Boekaerts y M. Pintrich (eds.), Handbook of Self- Regulation, San Diego: Academic Press, 631-650.

Stahl, R. J. y Murphy, G. T. (1981). The domain of cognition: An alternative to Bloom's Cognitive domain within the framework of an information processing model. (ERIC Document Reproduction Service No ED208511)

Thomson, P. y Sefton Green, J. (2011), Researching Creative Learning. Methods and issues, London/ New York: Routledge.

Wallace, B. et alii. (2012), "TASC: Thinking Actively in a Social Context. A Universal Problem-Solving Process. A Powerful Tool to Promote Differentiated Learning Experiences", Gifted Education International, 28, 1, 58-83. 